\title{
Development of a Robotic
} Endoscope Automated via Laryngeal Imaging for Tracheal Intubation (REALITI)

\section{Conference Paper}

Author(s):

Boehler, Quentin (1); Hofmann, Phyllis; Gage, D.; Gehring, A.; Chautems, Christophe; Biro, Peter; Nelson, Bradley (1)

Publication date:

2019-06

Permanent link:

https://doi.org/10.3929/ethz-b-000393102

Rights / license:

In Copyright - Non-Commercial Use Permitted 


\title{
Development of a Robotic Endoscope Automated via Laryngeal Imaging for Tracheal Intubation (REALITI)
}

\author{
Q. Boehler ${ }^{1}$, P. Hofmann, D. Gage ${ }^{2}$, A. Gehring, C. Chautems ${ }^{1}$, \\ P. Biro ${ }^{3}$, B.J. Nelson ${ }^{1}$
}

${ }^{1}$ Multi-Scale Robotics Lab, ETH Zurich

2 University of Zurich

${ }^{3}$ Institute of Anesthesiology, University Hospital Zurich qboehler@ethz.ch, peter.biro@usz.ch

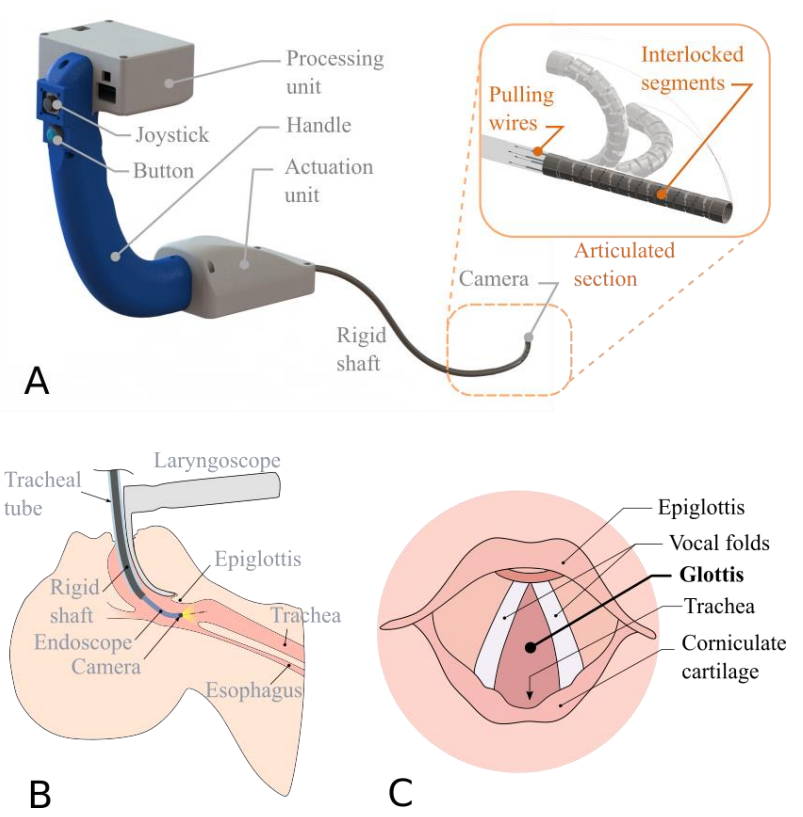

Fig. 1 Tracheal intubation with robotic assistance. A: REALITI device, B: Assisted tracheal intubation, $\mathrm{C}$ : Anatomy of the larynx from the endoscopic camera view.

\section{INTRODUCTION}

From general anesthesia to emergency medical services (EMS), tracheal intubation (TI) is considered to be the worldwide gold standard to secure the airway of a patient in need of respiratory assistance [1]. State-of-the-art TI consists of placing a tracheal tube (TT) into the trachea of the patient while lifting their jaw with a laryngoscope. This approach is called "direct-laryngoscopy" as it provides a direct view on the glottis, which is the entrance of the trachea. Inability to perform TI in time or TT misplacement can result in severe morbidity and mortality caused by asphyxia, hypoxemia, and pulmonary aspiration [2]. These casualties occur more frequently in EMS situations where TI is performed under difficult and stressful circumstances [3]. Moreover, anatomical factors or systemic conditions can make TI more difficult even for an experienced anesthesiologist in a hospital setting [4]. Alternatives to alleviate difficulties of direct-laryngoscopic TI have been established and include video-laryngoscopes or flexible endoscopes used to guide the TT [5]. However, when performed manually, a successful TI still relies solely on the dexterity and experience of the physician to first recognize key anatomical features such as the glottis, and then to steer the TT into the trachea. Our aim is to develop a robotic assistance for TI for these two critical aspects of the procedure using a Robotic Endoscope Automated via Laryngeal Imaging for Tracheal Intubation (REALITI). This work is focused on the development of a first prototype of the REALITI device, and its use in performing an assisted TI on a standard intubationtraining manikin.

\section{MATERIALS AND METHODS}

The REALITI is a handheld device comprised of a motorized 2-degrees-of-freedom endoscope, a handle, and a processing unit, as depicted in Fig. 1A. The articulated section located at the distal end of a rigid shaft acts as a guide for the TT. An endoscopic camera placed at its tip provides a constant video feedback of the distal end of the endoscope to an external monitor wired to the device.

To perform a TI with the device, the endoscope is inserted into the patient's mouth and moved forward manually towards the trachea (see Fig. 1B and C). The TT is then fed over the endoscope, and the device is removed from the patient's mouth while the TT is held in place. The device is intended to operate in two modes when being inserted into the trachea; (1) a manual mode where the bending motion of the endoscope is manually controlled by the user via a joystick, (2) an autonomous mode where the endoscope is automatically steered toward the glottis. Switching between the modes is triggered by the user via a button. Both the joystick and the button are located on the front of the handle and can be operated by the user with the thumb while moving the whole device.

The prototype of the device developed within the scope of this work integrates a customized, innovative design for the articulated section. This section consists of 24 interlocked segments cut from a $5.5 \mathrm{~mm}$ stainless steel rod using a micro-waterjet fabrication process. The outer diameter of the endoscope shaft is suitable for the positioning of standard TTs (6.5 to $9.0 \mathrm{~mm}$ inner diameter). The endoscope is steered by two pairs of antagonistic pulling wires attached to an actuation unit comprised of two DC-motors (1000:1 HPCB 6V dual- 
shaft, Pololu Co.). A processing unit (Odroid-XU4, Hardkernel Ltd.) with a Linux OS (Ubuntu 18.04) generates the control signals for the motors in both modes, and detects the anatomical features within the camera image (MD-VBS20904L-120-01, Misumi Electronics Co.). The user inputs coming from the joystick are used as the reference signals for a PID velocity controller for the two bending directions of the endoscope. A visual servo-control approach [6] is implemented to minimize the distance between the image center and the centroid of the detected glottis, when the autonomous mode is triggered by the user. Both controllers are tuned to ensure a smooth and stable motion of the endoscope within the two operating modes. The maximum speed of the distal orientation is limited to $60 \mathrm{deg} / \mathrm{s}$ in order to provide ergonomic handling of the device.

The detection of the anatomical features is performed using Haar Featured-based Cascade Classifiers [7] implemented in Python using the OpenCV library [8]. Four key features depicted in Fig. 2 are detected, and the recognition training is performed using 500 to 700 positive images and 500 to 900 negative images for each feature.

A dedicated user interface depicted in Fig. 3 is displayed on an external monitor and is comprised of the tip camera image, the detected anatomical features, the state of the tracking, the configuration of the endoscope, and the activated mode. Depending on the detected anatomical features and the recorded history, general instructions are also given, e.g. "Move the device to the left", "Go forward".

\section{RESULTS}

The initial testing of the endoscope manual control was first performed in an open environment. The endoscope could be controlled in a stable manner without overshoot for orientations of up to $60^{\circ}$ around its central axis and in all directions, which would be sufficient to perform a standard TI on a human patient.

When using the device to perform the TI on a manikin, the trachea was successfully entered using both operating modes and without prior training of the user in standard TI procedures. The autonomous mode could be used in the first stage of the procedure, i.e. right after the initial detection of the glottis, or when closer to the trachea, without further need to adapt the visual controller parameters. The anatomical features were detected at 200 $\mathrm{Hz}$ with the tip camera running at $30 \mathrm{fps}$, which is compatible with the required dynamic of the procedure. When first inserting the device into the oral cavity, the corniculate cartilage and/or the posterior commissure of the larynx are encountered (Fig. 2A). When moving toward the larynx, the glottis should be detected (Fig. 2B). Successful intubation occurs when the tip of the device has entered the subglottic trachea after passing the vocal folds (Fig. 2C).

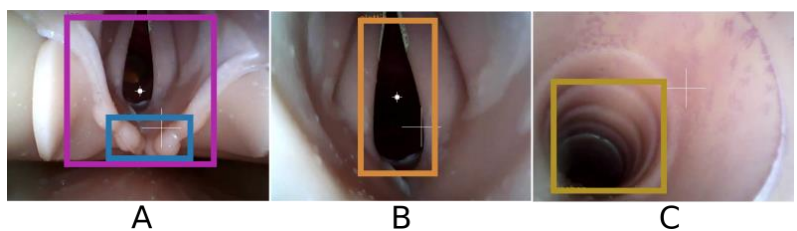

Fig. 2 Detected anatomical features in an intubation manikin along a TI with the REALITI device. A: Larynx (purple) and corniculate cartilage (blue), B: glottis, C: trachea.

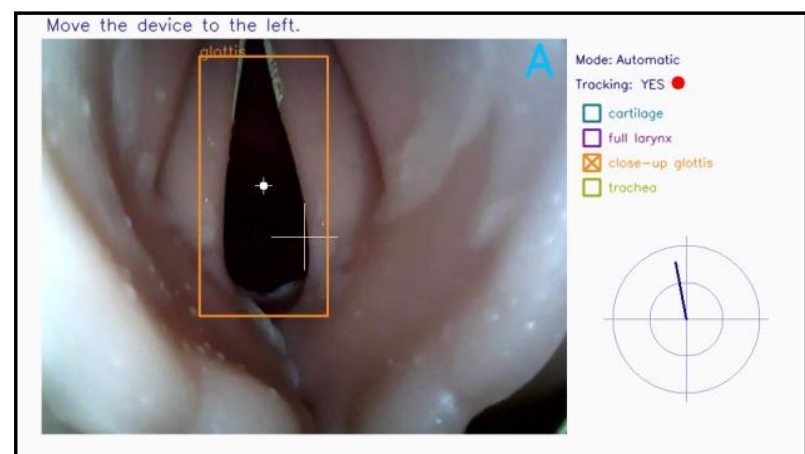

Fig. 3 User interface of the REALITI device. The state of the endoscope is represented as a line within a circular target indicating the azimuthal direction (angle of the line) and the amplitude of the direction (length of the line).

\section{DISCUSSION}

Our device is the first robotic endoscope dedicated to TI, which can assist the procedure by detecting features within the endoscopic image and by automated steering of the device toward the trachea. In this work, a prototype was developed to perform both manual and automated intubation on a training manikin. Results showed a distinct detection of the glottis and a stable motion in both modes. The next steps will include the development of a preclinical prototype for ex vivo testing and the implementation of the glottis detection on in vivo endoscopic images.

\section{REFERENCES}

[1] Thomas EBM, Moss S. Tracheal intubation Anaesthesia \& Intensive Care Medicine 2017; 15: 5-7

[2] Apfelbaum JL et al. Practice guidelines for management of the difficult airway: an updated report by the American Society of Anesthesiologists Task Force on Management of the Difficult Airway. Anesthesiology. Feb 2013;118(2):251-70.

[3] Pepe PE, Roppolo LP, Fowler RL. Prehospital endotracheal intubation: elemental or detrimental? Crit Care, 2015. 19: p. 121.

[4] Glick DB, Cooper R, Ovassapian A. (Eds.). (2012). The difficult airway: An atlas of tools and techniques for clinical management. Springer Science \& Business Media.

[5] McCluskey K, Stephens M. Alternative techniques for tracheal intubation. Anaesthesia \& Intensive Care Medicine 2017; 18: 163-167

[6] Chaumette F, Hutchinson S. Visual servo control. II Advanced approaches [Tutorial]. IEEE Robotics \& Automation Magazine, 2007 Mar;14(1):109-118.

[7] Viola P, Jones M. (2001). Rapid object detection using a boosted cascade of simple features. CVPR (1), 1, 511-518. OpenCV: Cascade Classifier Training. Retrieved from https://docs.opencv.org/3.4.3/dc/d88/tutorial _traincascade.html 